\title{
Investigation of HVAC Operation Strategies for Office Buildings During COVID-19 Pandemic
}

\author{
Cary A. Faulkner ${ }^{\mathrm{a}}$, John E. Castellini Jr. ${ }^{\mathrm{a}}$, Wangda Zuo ${ }^{\mathrm{a}, \mathrm{b}}$, David M. \\ Lorenzettic $^{\mathrm{c}}$, Michael D. Sohn ${ }^{\mathrm{c}}$ \\ ${ }^{a}$ Department of Civil, Environmental and Architectural Engineering, University of \\ Colorado Boulder, UCB 428, Boulder, 80309, CO, U.S.A. \\ ${ }^{b}$ National Renewable Energy National Laboratory, Golden, 80401, CO, U.S.A. \\ ${ }^{c}$ Energy Analysis and Environmental Impacts Division, Lawrence Berkeley National \\ Laboratory, 1 Cyclotron Road, Berkeley, 94720, CA, U.S.A.
}

\section{Abstract}

To minimize the indoor transmission of contaminants, such as the virus that can lead to COVID-19, buildings must provide the best indoor air quality possible. Controlling indoor air quality is largely achieved by running the building's HVAC system to dilute any concentration of indoor contaminants. However, doing so has practical downsides on the HVAC operation that are not always quantified in the literature. This paper develops a temporal simulation capability that is used to investigate the indoor virus concentration and operational cost of an HVAC system for two mitigation strategies: 1) supplying $100 \%$ outdoor air into the building and 2) using different HVAC filters, including MERV 10, ASHRAE minimum-recommended MERV 13, and highly rated HEPA filtration. These strategies are applied to a hypothetical 
medium office building consisting of five occupied zones and located in a cold and dry climate. We modeled the building using the Modelica Buildings library and developed new models for HVAC filtration and virus transmission to evaluate COVID-19 scenarios. We show that the ASHRAE-recommended MERV 13 filtration reduces the average virus concentration by about $10 \%$ when compared to MERV 10 filtration, with negligible additional operational cost. In contrast, the use of $100 \%$ outdoor air or HEPA filtration reduces the average indoor concentration by about an additional 3\% compared to MERV 13 filtration, but significantly increases building operational cost. This is due to the significant increase in energy consumption when supplying $100 \%$ outdoor air and the higher cost of purchasing a HEPA filter.

Keywords: COVID-19, Indoor Air Quality, Building Energy, Modelica.

\section{Introduction}

The COVID-19 pandemic has increased the need for buildings to improve their indoor air quality to help reduce the risk of infection from airborne transmission. A recent study [1] found that all 318 identified outbreaks of three or more COVID-19 cases in China occurred in indoor environments. Another study [2] found an outbreak of 55 cases among 81 attendees of in-person exercise classes at an indoor facility in Chicago, IL. It was also 
found that the air-conditioning system played a large role in the infection of patrons inside a restaurant in Guangzhou, China [3]. These studies demonstrate the significant risk of indoor infection. As a result, organizations, such as ASHRAE in January 2021 [4], provided recommendations for building operation during the pandemic to improve indoor safety. Included were recommendations for the heating, ventilation, and air conditioning (HVAC) system operation, such as providing necessary ventilation and using filters that achieve minimum efficiency reporting value (MERV) 13 rating or better. The recommended strategies have been shown to be helpful in improving indoor air quality, but their impacts on the HVAC system operation, such as energy consumption, are not quantified and need to be investigated further.

Previous literature tried to study and compare strategies to improve indoor air quality to better understand this issue. Ben-David and Waring [5] compared the use of different ventilation rates and HVAC filter ratings using a holistic cost function approach to consider indoor air quality $\left(P M_{2.5}\right.$ and ozone concentrations), energy consumption, and filter cost tradeoffs. They found that improving filtration tended to have a more significant effect than increasing ventilation. Azimi and Stephens [6] studied risk reduction of influenza virus and associated operational costs for different filter ratings and 
equivalent levels of outdoor air ventilation. In addition, they found filtration improved risk reduction with lower costs compared to increased ventilation. More recently, Pease et al. [7] investigated the effect of filtration, air change rate, and outdoor air fraction on the concentration of COVID-19 virus and probabilities of infection in a multi-room building. Similarly, they found filtration was the best method for reducing virus concentration. The study also suggested that although increasing outdoor air rate or air change rate is proved to be beneficial, they should be used with caution. For example, increasing the outdoor air rate can increase the heating or cooling energy used by the HVAC system due to the temperature difference between the indoor and outdoor air.

Although significant progress has been made in the literature, further improvements can be made. First, current studies lack detailed modeling of the operation and control of the HVAC system, for example by assuming constant ventilation rates. As a result, the dynamic effects of the HVAC system, which are critical for real system operation, are lost. For example, the controls of the outdoor air damper and supply fan can affect the dilution and removal rate of indoor virus concentration as well as the HVAC energy consumption. Also, many studies evaluate risk with steady-state concentrations 
and constant occupancy, while both these values are dynamic in practice. Finally, while some of these studies perform annual simulations, they do not compare the indoor air quality and their associated operational cost during different times of the year. The effect of outdoor conditions can determine the optimal operating strategy, which may vary over the course of the year.

To address this gap, we use an equation-based, object-oriented modeling language (Modelica) to develop a detailed model of an HVAC system and enable temporal analyses. For example, Fu et al. [8] used Modelica-based models to study cooling systems in data centers and investigate their performance during normal and emergency conditions. Huang et al. [9] used Modelica-based models to study control related faults for chiller and boiler plants. Tian et al. [10] used computational fluid dynamics coupled with Modelica to optimize thermostat placement in an office room based on thermal comfort and energy consumption. The efficient dynamic modeling offered by Modelica language, as well as the availability of the Modelica Buildings library [11, 12], were enabling features to these studies. Additionally, our developed models for HVAC filters and virus transmission, to our knowledge, have not yet been created using a Modelica-based platform.

The remainder of this paper is organized as follows. First, a review of 
HVAC operation strategies to mitigate infection of airborne virus indoors is presented in Section 2. Next, the implementation of new models for HVAC filters and virus transmission into an existing Variable Air Volume (VAV) system model for a medium office building is detailed in Section 3. Then, results for virus concentration and energy consumption for the different strategies are shown and the combined results are analyzed in Section 4 . Finally, the paper is concluded in Section 5.

\section{Review of HVAC Operation Strategies to Mitigate Indoor Disease Transmission}

There are several methods to improve indoor air quality by HVAC operation, each with different benefits and drawbacks. Guo et al. [13] summarized and compared HVAC operation guidelines during the COVID-19 pandemic for buildings in different countries. Based on this review, common strategies include: 1) increasing ventilation rates of outdoor air as high as possible, 2) running the HVAC system for longer periods to flush out lingering virus, and 3) improving filtration of recirculating air. Increasing the supply rate of outdoor air can dilute the indoor concentration of virus without the need of purchasing and maintaining new equipment, as well as without increasing 
the system pressure drop in the air handling system. However, increasing the supply flow rate of outdoor air can significantly increase cooling or heating energy consumption when the outdoor air temperature differs greatly from the room temperature setpoint. It may also sacrifice the thermal comfort as most HVAC systems are not sized for high outdoor airflow rates. Secondly, increasing the runtime of the HVAC system, for example running the system longer before or after occupants arrive, can help flush out virus lingering in the room. However, this approach has limits, especially considering the increased likelihood of virus emission and infection during regular operational hours when there are more occupants. Finally, improving the filtration of recirculating air can reduce virus concentration without increasing heating or cooling energy consumption due to increased ventilation. However, the airflow resistance caused by HVAC filters increases the pressure drop through the air handling system, which can increase fan energy consumption and/or reduce the system flow rate [14]. Furthermore, there are associated financial costs of purchasing and installing the HVAC filters, as well as replacing the filter after it accumulates particles over time.

This review suggests that mitigation strategies might benefit, or differ, if an evaluation considered the typical change in occupancy, as well as cooling 
and heating loads, over the course of a day. Thus, this paper studies the strategies of supplying $100 \%$ outdoor air and using efficient HVAC filters, since these strategies can be used to improve the indoor air quality throughout the day. There are many HVAC filters that are often defined by their MERV rating, which describes their ability to filter particles of different sizes [15]. ASHRAE recommends to use filtration that achieves at least MERV 13 rating during the pandemic due to their ability to filter at least $85 \%$ of virus droplets with diameter between 1-3 $\mu m$ [16]. The most efficient filters are known as high-efficiency particulate absorbing (HEPA) filters, which exceed MERV 16 rating and filter $99.97 \%$ of virus droplets greater than $0.3 \mu m$ [17]. Thus, this paper investigates the use of MERV 13 and HEPA rated filters, representing the minimum recommended rating for use during the pandemic and the maximum achievable rating. These strategies are evaluated against MERV 10 filtration, which filters at least $50 \%$ of virus droplets with diameter between 1-3 $\mu m$ [15] and may be used in older buildings. It should be noted the $100 \%$ outdoor air strategy is assumed to use a MERV 10 filter, since buildings use HVAC filters to filter outdoor air as well. 


\section{Model Implementation and Verification}

For the temporal simulations, we first developed a temporal simulation of a variable-air-volume (VAV) HVAC supply system for a typical medium office building. We based the building model along a prototype provided in the Modelica Buildings library [18]. For our simulations, we developed modules to supplement the prototype to represent HVAC filtration and virus transmission. In this section, we describe the features of the model of the medium office building and our verification of the new models to support this study. Finally, we describe the whole building model with the new modules incorporated.

\subsection{Overview of the Building System}

This building was based on the DOE commercial reference medium office building [19], with focus on the bottom floor building prototype. The floor contains five zones, including a core zone and four perimeter zones, as shown in Figure 1. These zones are assumed to be well-mixed in the model, with volumes of 2,698 $\mathrm{m}^{3}$ for the Core Zone, $569 \mathrm{~m}^{3}$ for the North and South zones, and $360 \mathrm{~m}^{3}$ for the East and West zones. We have a central air handling unit with heating and cooling coils with VAV terminal boxes containing 
reheat coils in each zone. The HVAC system is sized for the location of Denver, Colorado. Cooling is provided using chilled water with coefficient of performance of 5 , which is defined as the ratio of the rate of cooling provided to the input electrical power. Heating is provided using a hot water system with efficiency of 0.8 , which is the ratio of the rate of heating provided to the input required power. The system is controlled based on the control sequence VAV 2A2-21232 from the Sequences of Operation for Common HVAC Systems described in [20].

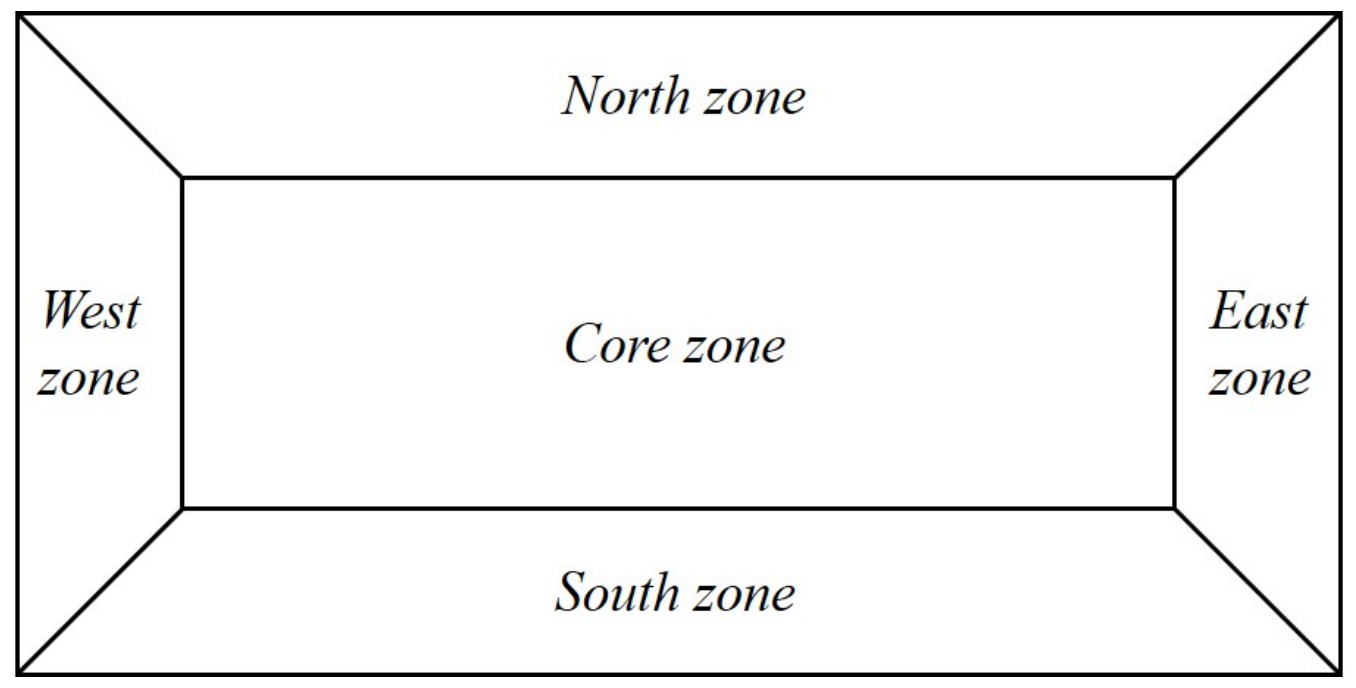

Figure 1: Floor layout of the medium office building with five zones. 


\subsection{Implementation and Verification of New Models}

The development and verification of the new models to support this study are detailed next. First, the new HVAC filter component model is described, followed by the models for virus generation and decay.

\subsubsection{HVAC Filter Model}

An HVAC filter model was developed to support the work for this paper, since such a model was not included in the original VAV system model. The model includes two main components: removal of virus based on a defined efficiency and static pressure drop depending on the mass flow rate and defined nominal flow conditions.

The removal of virus can be described as:

$$
c_{\text {out }}=\left(1-\eta_{\text {filter }}\right) c_{\text {in }},
$$

where $c_{\text {out }}$ is the virus concentration exiting the filter, $\eta_{\text {filter }}$ is the filter

removal efficiency in terms of percentage of virus removed, and $c_{i n}$ is the virus concentration entering the filter. The filter efficiency can be between 0-100\%, where $\eta_{\text {filter }}=100 \%$ describes a filter that completely removes all virus in the airflow. 
The removal of virus for the HVAC filter model was verified with a simple unit study, by supplying air with concentration $c_{0}$ to a room initially virus free for different filter efficiencies. The case was run for 500 seconds, when the concentrations approach their equilibrium values. The results in Figure 2 show the normalized concentrations approach their expected steady-state values of $\left(1-\eta_{\text {filter }}\right) c_{0}$, since the filter removes a fraction of $\eta_{\text {filter }}$ from the supply flow with concentration $c_{0}$.

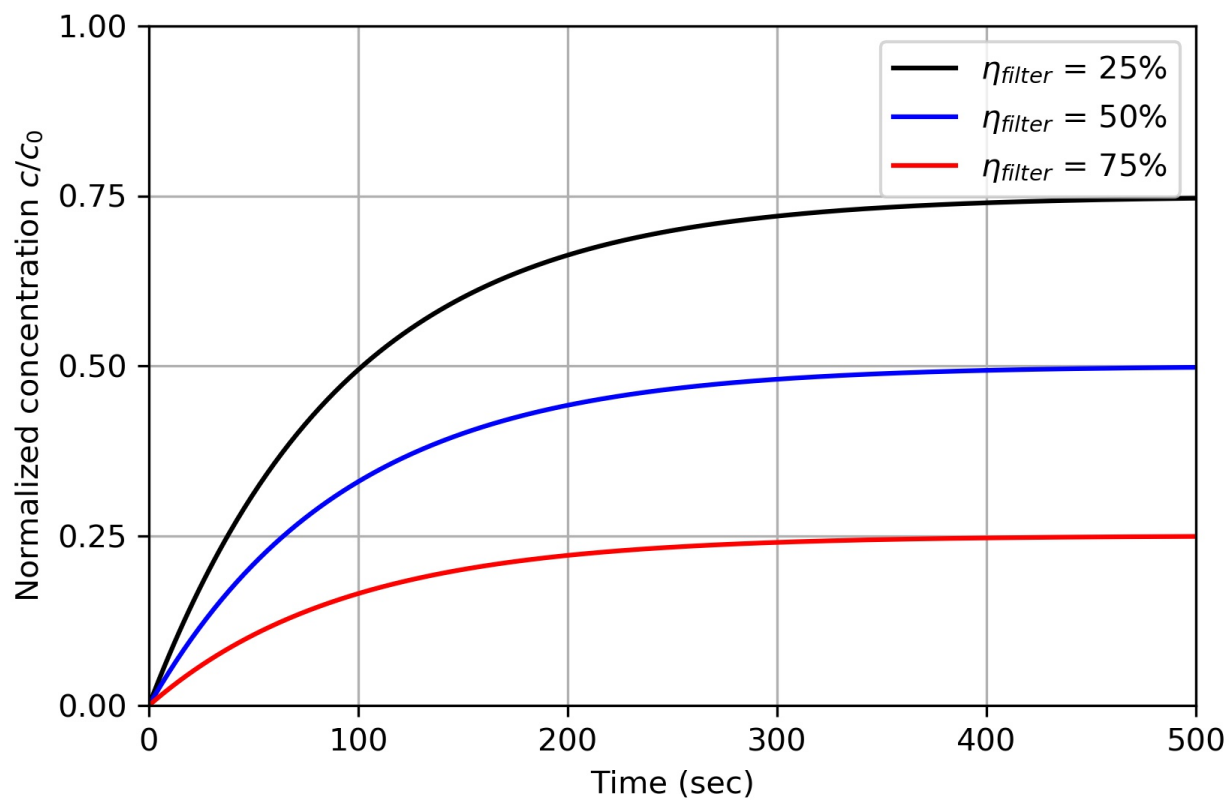

Figure 2: Normalized room virus concentrations over time with different HVAC filter efficiencies. 
Next, the static pressure drop caused by the resistance of the filter is:

$$
\Delta p_{\text {filter }}=k_{\text {filter }} \dot{m}_{\text {filter }}^{2},
$$

where $\Delta p_{\text {filter }}$ is the static pressure drop caused by the filter, $\dot{m}_{\text {filter }}$ is the mass flow rate of air though the filter, and $k_{\text {filter }}$ is:

$$
k_{f i l t e r}=\frac{\Delta p_{\text {nom }}}{\dot{m}_{\text {nom }}^{2}},
$$

where $\Delta p_{\text {nom }}$ is the nominal pressure drop at the nominal mass flow rate, $\dot{m}_{\text {nom }}$. These two values are inputs to the filter model. The quadratic relation between static pressure drop and mass flow rate can be approximated using the Bernoulli equation [21] and captures the general trend from experimental data [15]. It should be noted that the filter pressure drop increases over time as the filter collects particles [22], but the nominal pressure drop was assumed to be constant in this study for simplicity.

The pressure drop as a function of flow rate for the HVAC filter model was verified by supplying air at rates of $0.5 \mathrm{~kg} / \mathrm{s}-1.5 \mathrm{~kg} / \mathrm{s}$ with different filter nominal pressure drops. The nominal mass flow rate for the filter was held constant at $1.0 \mathrm{~kg} / \mathrm{s}$ for all the cases. The verification results are shown in Figure 3. 


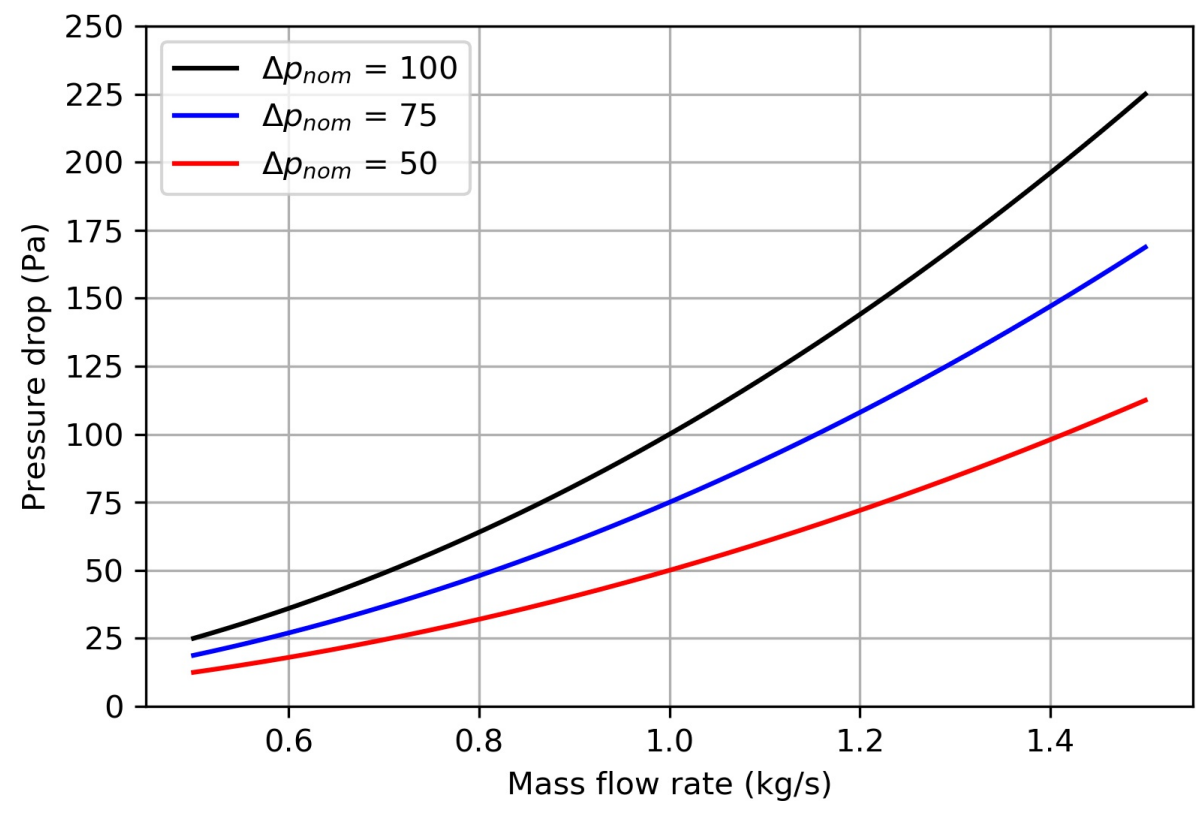

Figure 3: Filter pressure drop vs mass flow rate for different nominal pressure drops.

The results show the quadratic relation between pressure drop and mass flow rate, as expected based on Equation 2. It can also be seen that the pressure drops for the three cases pass through their nominal values at the nominal mass flow rate of $1.0 \mathrm{~kg} / \mathrm{s}$.

The settings used for the filters used in this study are shown in Table 1. The filter efficiencies come from ASHRAE technical resources [16] and the nominal pressure drop values are chosen based on data for MERV 10 [23], 
MERV 13 [24], and HEPA [25] filters based on the nominal flow rate of the studied system.

\begin{tabular}{|c|c|c|}
\hline Filter & Nominal Pressure Drop (Pa) & Efficiency \\
\hline MERV 10 & 17 & $50 \%$ \\
\hline MERV 13 & 37 & $85 \%$ \\
\hline HEPA & 250 & $99.97 \%$ \\
\hline
\end{tabular}

Table 1: HVAC filter simulation settings.

\subsubsection{COVID-19 Virus Modeling}

Additionally, models for the generation and decay of virus in the rooms were developed for this study. First, we simulated the presence of one "sick" person in each zone working from 9:00 AM to 5:00 PM, Monday through Friday throughout the year. The purpose of the presence of sick people throughout the year was to study the concentrations and effect of strategies in all the zones during the different seasons. Generation of virus was described in terms of quanta emission rates, where a quantum is the dose of airborne droplet nuclei expected to cause infection in $63 \%$ of susceptible people [26]. A typical value for quanta emission of 100 quanta/hr was used for the majority 
of this study based on literature [7, 26], although different quanta emission values are considered in Section 4.1.3 to compare the risk of infection for different emission rates. The virus was generated directly in each well-mixed zone when the sick people were present based on the quanta emission rate.

The viral decay in the room due to gravitational settling and the death of airborne viruses is modeled based on Equation 4, which has been used in literature to model viral decay in well-mixed zones [7]. This is described as:

$$
\dot{c}_{\text {decay }, z o n e}=k_{\text {decay }} c_{\text {zone }},
$$

where $\dot{c}_{\text {decay,zone }}$ is the rate of viral decay in the zone, $k_{\text {decay }}$ is a defined constant rate of viral decay, and $c_{z o n e}$ is the virus concentration in the zone.

The decay model was verified by examining the viral decay in a room with initial concentration $c_{0}$. There were no other means to produce or remove virus, other than loss due an holistic decay rate. For this case, the virus concentration in the room can be derived analytically as:

$$
c(t)=c_{0} \exp \left(-k_{\text {decay }} t\right)
$$

where $c(t)$ is the transient virus concentration in the room, $c_{0}$ is the initial virus concentration, $k_{\text {decay }}$ is the viral decay rate, and $t$ is time. The results 
for virus concentration over time with different viral decay rates are shown in Figure 4. It can be seen that the virus concentration decays exponentially and decays faster for larger values of $k_{\text {decay }}$.

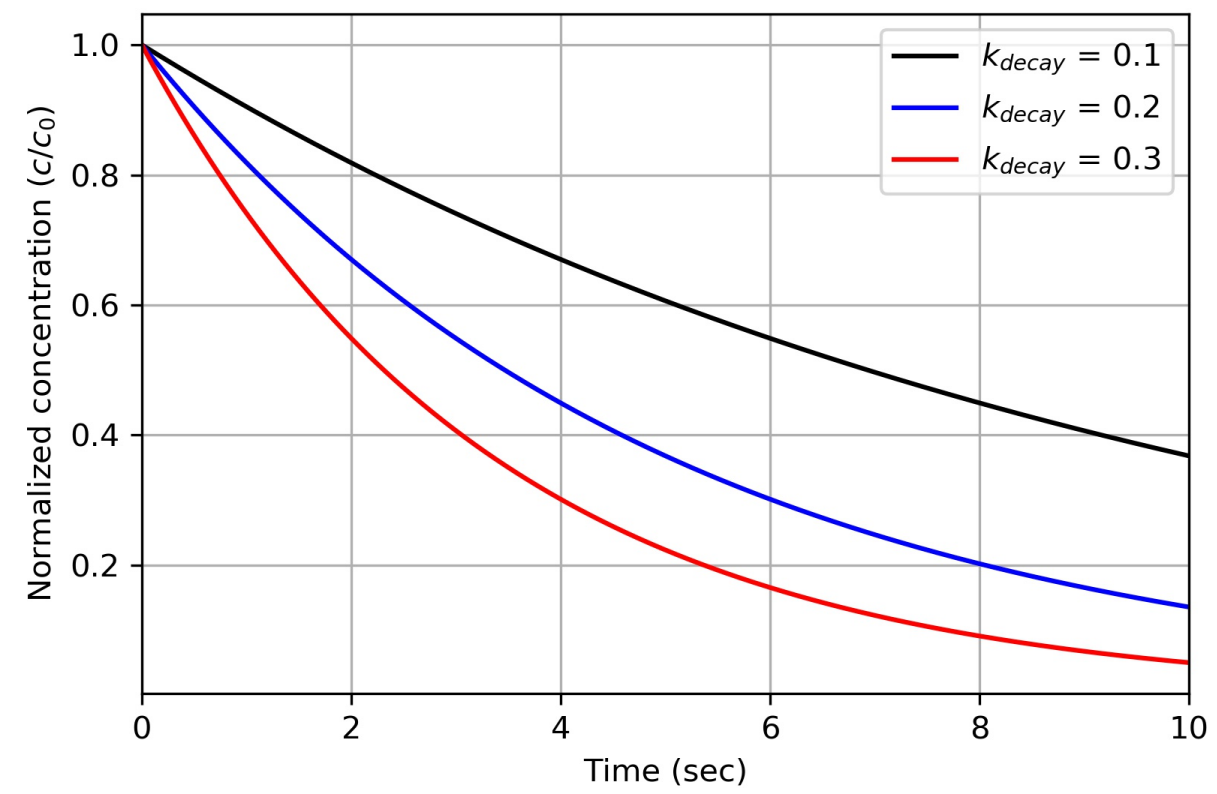

Figure 4: Normalized concentration over time with different decay rate values.

To quantify the impact of the virus concentrations, risk of infection is calculated using the Wells-Riley approach, which determines this risk based on the amount of virus inhaled by an occupant. Risk of infection is calculated as: 


$$
R(t)=1-\exp \left(-I R \int_{t_{0}}^{t} c(t) d t\right)
$$

where $R(t)$ is risk of infection in terms of percentage, $I R$ is the volumetric inhalation rate of air for an occupant, and $\int_{t_{0}}^{t} c(t) d t$ is the integral of virus concentration in the room with respect to time since initial time $t_{0}$. The predicted number of infections, $R_{0}$, can be calculated based on the risk, $R$. The predicted number of infections over time, $R_{0}(t)$ is calculated accounting for the variable occupancy in the zone for this study. This is done by calculating $R_{0}(t)$ for a given time interval when the occupancy is constant and adding the predicted number of infections calculated from the previous time interval. This can be described as:

$$
R_{0, T}(t)=S_{T}\left[1-\exp \left(-I R \int_{t_{0}}^{t} c(t) d t\right)\right]+R_{0, T-1}\left(t_{0}\right)
$$

where $R_{0, T}(t)$ is the predicted number of infections in the zone for time interval $T, S_{T}$ is the number of susceptible occupants in the zone during $T$, $t_{0}$ is the time at the beginning of interval $T$, and $R_{0, T-1}\left(t_{0}\right)$ is the predicted number of infections from the previous time interval, $T-1$, ending at time $t_{0}$. Susceptible occupants is determined as $S=N-1$, where $N$ is the number of occupants. This way $S$ does not account for the sick person, since they cannot 
infect themselves. Figure 5 shows the predicted number of infections for a sample day based on the virus concentration and occupancy. A sample time interval, $T$, is highlighted to show how $R_{0}$ is calculated based on the number of susceptible occupants and amount of inhaled virus during this time. At the beginning of this time interval, the slope of $R_{0}(t)$ initially increases due to the increase in occupancy as the susceptible occupants return from lunch. $R_{0}(t)$ then steadily increases as occupants constantly inhale virus in the zone. Finally, $R_{0}(t)$ quickly flattens out after this interval as occupants leave and the virus concentration decreases. For this study, the values for viral decay and inhalation rate were chosen to be $0.48 \mathrm{hr}^{-1}$ and $0.48 \mathrm{~m}^{3} / \mathrm{hr}$ based on literature [7]. 


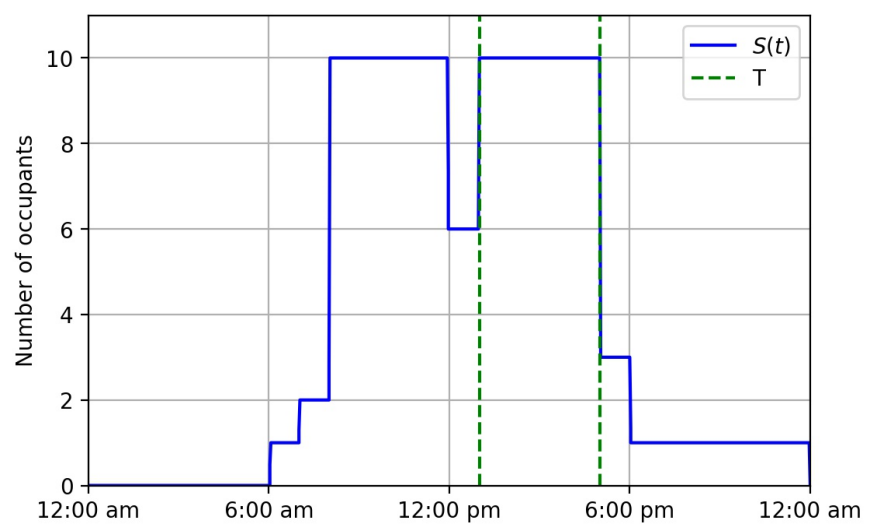

(a) Occupancy during the day.

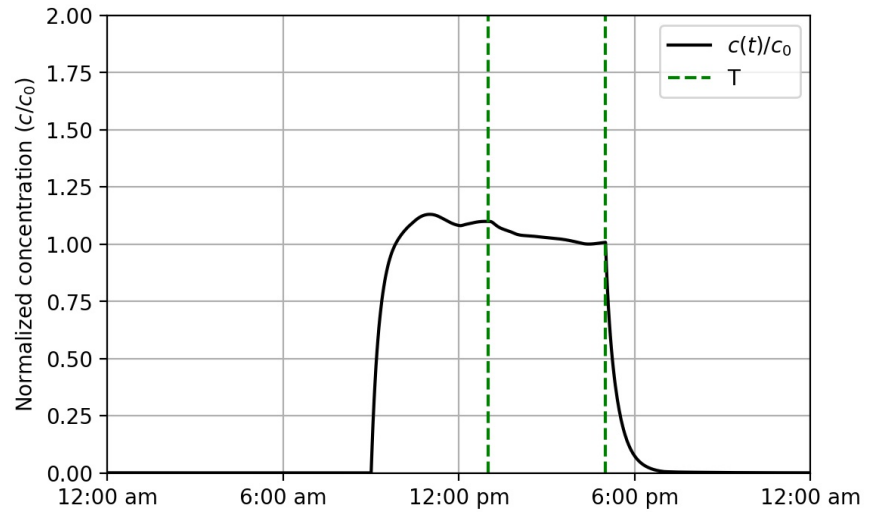

(b) Normalized virus concentration.

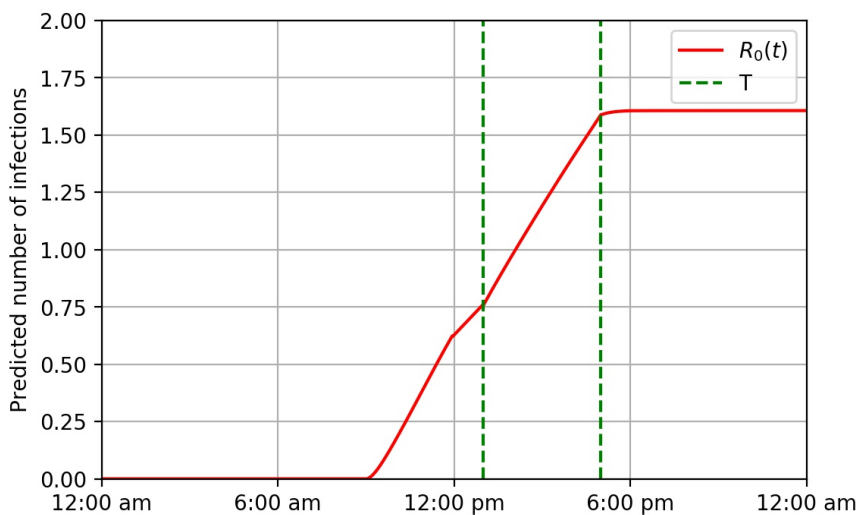

(c) Predicted number of infections.

20

Figure 5: Predicted number of infections based on the occupancy and virus concentration for a sample day. 


\subsection{Whole Building Model}

The newly developed models were added to the VAV system model to perform this study and the final Modelica model capability is shown in Figure 6. The entire model can be divided into four sections: (1) the multizone airflow model for the five zones, which includes the generation and decay of virus in the zones; (2) the VAV system model which includes the central air handling unit, as well as VAV terminal boxes and the return duct; (3) the control system which includes PI controllers for the heating and cooling coils, outdoor air economizer, and supply fan; and (4) the weather conditions, including dry bulb temperature, wind speed, and radiative exchange.

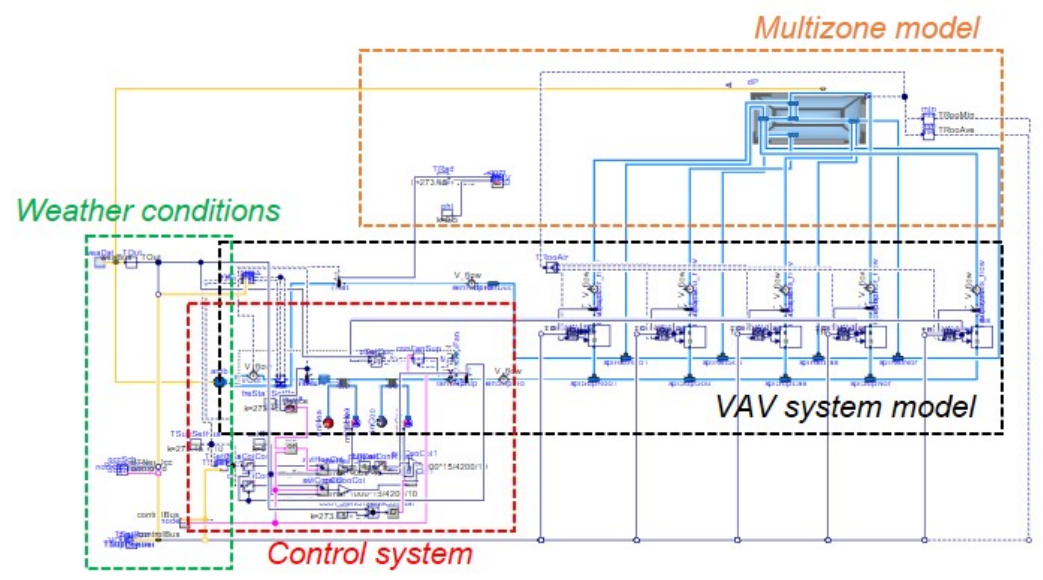

Figure 6: Modelica model of the medium office building. 


\section{Results and Discussions}

In this section, the results for indoor virus concentration are presented first, followed by the results for energy consumption. Finally, analysis of the combined results is performed to consider best overall strategies based on both indoor air quality and operational cost.

\subsection{Virus Concentration Results}

The virus concentration results are presented for different time scales in this section. First, the annual average virus concentration results in the five zones for the different strategies are presented. Next, the monthly average results are presented to show how the concentrations vary throughout the year. It is worth noting that the annual and monthly averages only account for the concentrations during occupied hours. Finally, results from a sample day are presented including risk analysis based on predicted number of infections.

\subsubsection{Annual Virus Concentration Results}

The results of the annual-average virus concentration by four different strategies in five different zones are shown in Figure 7. The indoor virus concentration results in this figure and throughout this section are normalized 
by $c_{0}$, which is the building average virus concentration for the MERV 10 case (first blue bar on the left in Figure 7). The results show that the strategies of supplying 100\% outdoor air and use of HEPA filtration provide the lowest virus concentrations, since they both essentially provide $100 \%$ clean air to the zones. These strategies reduce the annual building-average virus concentration by about $13 \%$ compared to use of MERV 10 filtration. While use of MERV 13 filtration does not reduce the virus concentration as much as the two previously mentioned strategies, it still reduces the annual building-average virus concentration by about $10 \%$ compared to use of MERV 10 filtration. In terms of the concentrations in each of the zones, the Core zone has the lowest average concentration. In large part because it is the largest zone but has the same number of sick people as the other zones. The North zone has the highest concentrations for all the strategies due to having the lowest nominal flow rate based on the system sizing. This zone will be used for further analysis in Section 4.1.3. 


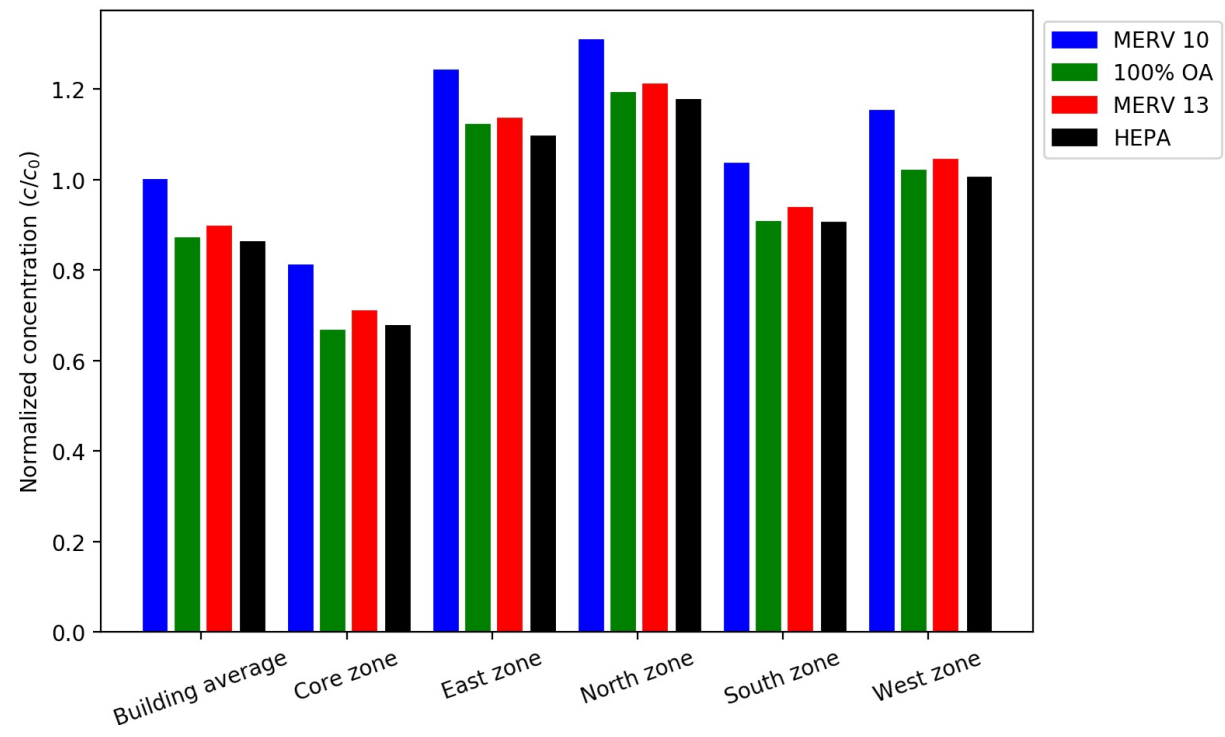

Figure 7: Annual-average, normalized virus concentration results for the different strategies and zones.

Figure 8 shows the variation of concentration for the four strategies during the day throughout the year. For each weekday, the concentration begins to increase at 9:00 AM when the sick people arrive, then decays quickly when they leave at 5:00 PM. The MERV 10 case reveals the increased concentration during the summer when the HVAC system tends to supply less outdoor air. The other cases do not show a significant variation during the year, since they are less sensitive to the outdoor air fraction due to their ability to remove virus efficiently. 


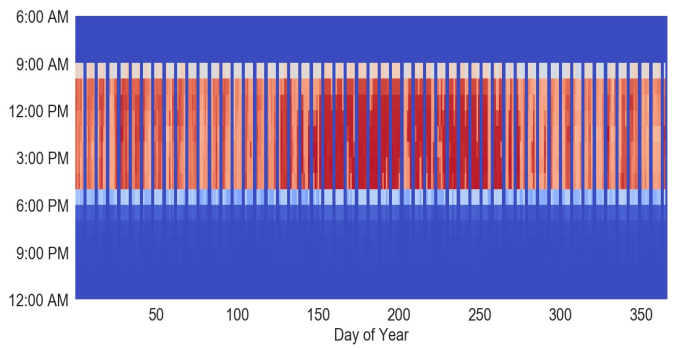

(a) MERV 10

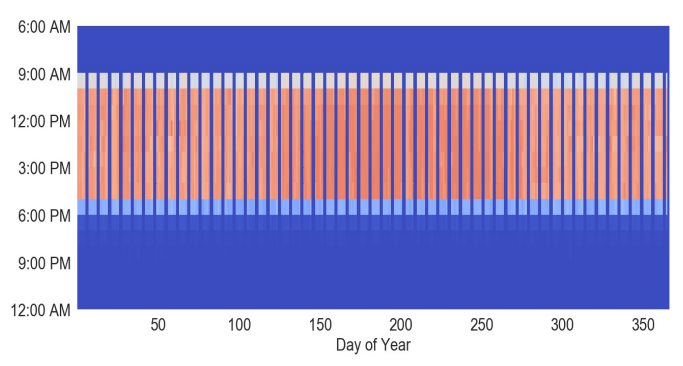

(c) MERV 13

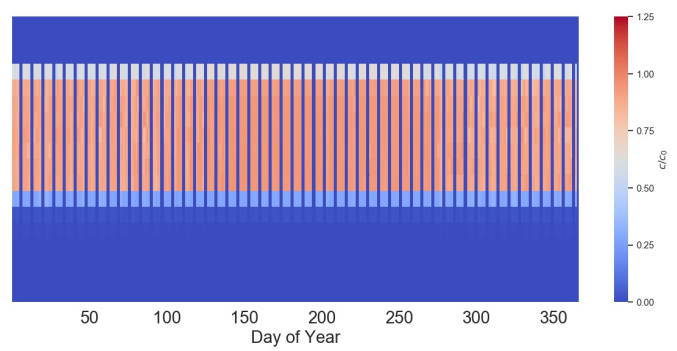

(b) $100 \%$ outdoor air

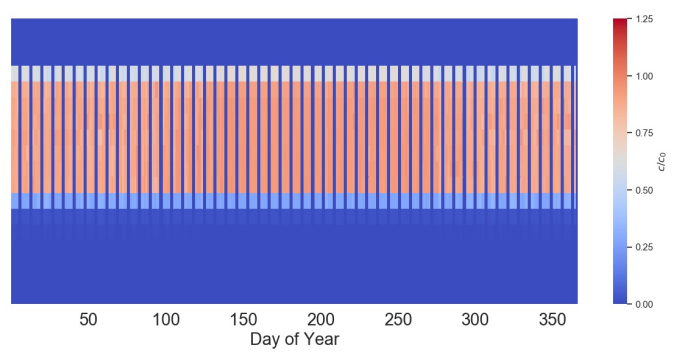

(d) HEPA

Figure 8: Heat maps showing the magnitude of normalized concentration during the days over the course of the year.

\subsubsection{Monthly Virus Concentration Results}

The monthly results for building-average virus concentration for the four strategies are shown in Figure 9. This further reveals the implications of changing seasons on the virus concentration for the different strategies. While the average concentrations for the use of $100 \%$ outdoor air and HEPA filtration vary slightly month-to-month, the MERV 10 case varies more due to its 
sensitivity to outdoor air fraction. Also, the variation of concentration for the MERV 13 case is more apparent in this figure, since it still does not supply as clean air as the $100 \%$ outdoor air and HEPA cases. The results show the lowest average virus concentrations for the MERV 10 and MERV 13 cases occur during April, October, and November when the weather is most mild and the HVAC system tends to supply more outdoor air. Similarly, the highest concentrations for these cases occur during the hot summer months when the system often supplies the minimum outdoor air. This reveals the advantages of the $100 \%$ outdoor air and HEPA filtration strategies, relative to the MERV 10 and MERV 13 cases, during the summer based on the virus concentration. The reduced advantages of these strategies during mild weather, based on the virus concentration, are also apparent. 


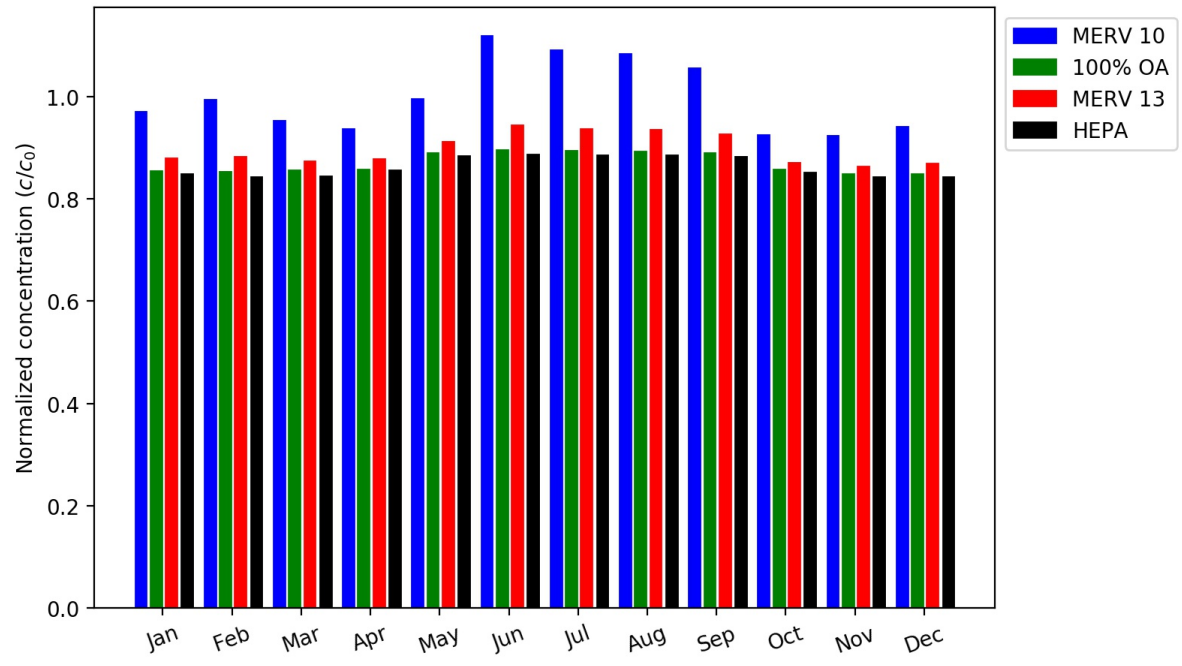

Figure 9: Monthly building-average virus concentration results for the different strategies.

\subsubsection{Sample Day Virus Concentration Results}

Transient results for virus concentration and risk in the worst zone (North Zone) for two sample days are shown in this section. The emission of virus depends on the individuals and their activities, for example if they are speaking or exercising. To capture that variation, three different virus generation rates ( $q=50$ quanta/hr, 100 quanta/hr, and 150 quanta/hr) were considered. These generation rates roughly span low, moderate, and higher activity of a sick person. 
First, virus concentration results for a hot summer day are shown in Figure 10. It shows the differences among the strategies when the filter cases use the minimum outdoor supply flow rate. All the strategies follow a similar trend throughout the day. The virus concentration increases at 9:00 AM when the sick people arrive, tends towards an equilibrium during the middle of the day, then sharply decreases when the sick people leave at 5:00 PM. The 100\% outdoor air and HEPA filtration strategies reduce the virus concentration for this day by up to $23 \%$ compared to the MERV 10 case. Use of MERV 13 filtration reduces the virus concentration by up to 17\% compared to MERV 10 filtration for this day. 


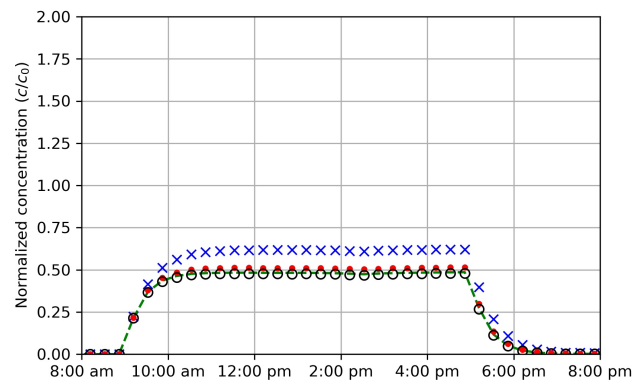

(a) $q=50$ quanta $/ \mathrm{hr}$

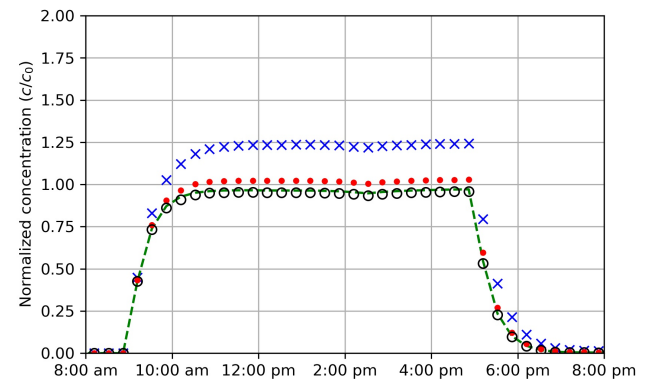

(b) $q=100$ quanta/hr

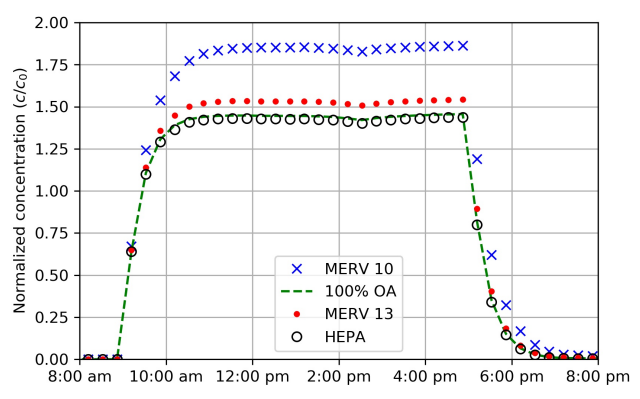

(c) $q=150$ quanta/hr

Figure 10: Normalized virus concentration in the worst zone for a hot day with different virus generation rates.

To better understand the implication of these virus concentrations, the predicted number of infections over time, $R_{0}(t)$, for this day are shown in Figure 11. The higher generation rates increase the predicted number of infections, as expected due to the higher virus concentrations. This is because the predicted number of infections accounts for the amount of virus inhaled 
by the susceptible occupants throughout the day. Both the occupancy and concentration vary throughout the day, so the predicted number of infections is calculated and summed for each hour to account for these dynamic effects. For example, $R_{0}(t)$ begins to increase at 9:00 $\mathrm{AM}$ as the concentration increases and susceptible occupants are exposed to the virus. The slope of $R_{0}(t)$ then decreases at 12:00 PM when occupants leave for lunch, but the slope increases again when they return at 1:00 PM. Finally, $R_{0}(t)$ flattens out at its final value when the virus concentration decays to zero at the end of the day.

Even for the most effective strategies, it is possible or even very likely that one infection will occur in this zone, based on the different generation rates shown in Figure 11. For example, $R_{0}$ reaches 0.75 at the end of the day for the lowest generation rate case with HEPA filtration. This suggests that there is a $75 \%$ chance that one person in the zone that day is exposed to a level that could result in an infection. For the medium and high generation rates, $R_{0}$ is reduced by about 0.40 and 0.50 , respectively, at the end of the day for both the HEPA and 100\% outdoor air cases compared to the MERV 10 case. MERV 13 filtration reduces $R_{0}$ by about 0.30 and 0.40 , respectively compared to MERV 10 filtration at the end of the day for the medium and 
high generation rates. While MERV 13 filtration is less effective compared to use of $100 \%$ outdoor air or HEPA filtration for the high generation rate on this day, the increased $R_{0}$ of about 0.10 is still relatively small.

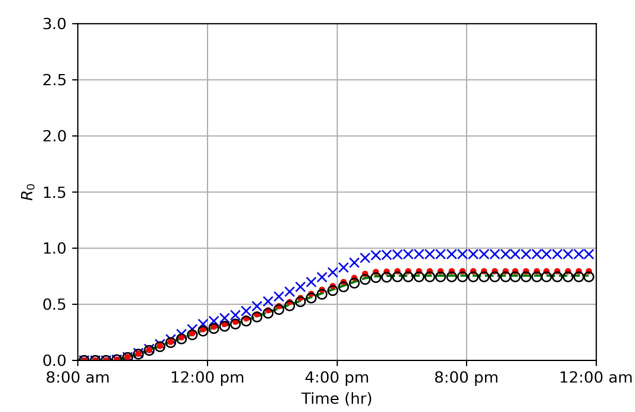

(a) $q=50$ quanta/hr

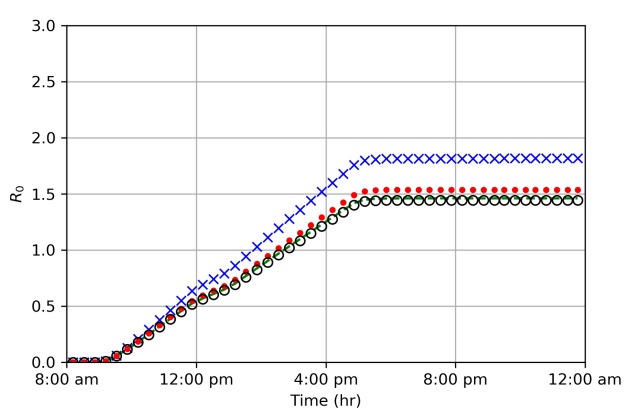

(b) $q=100$ quanta $/ h r$

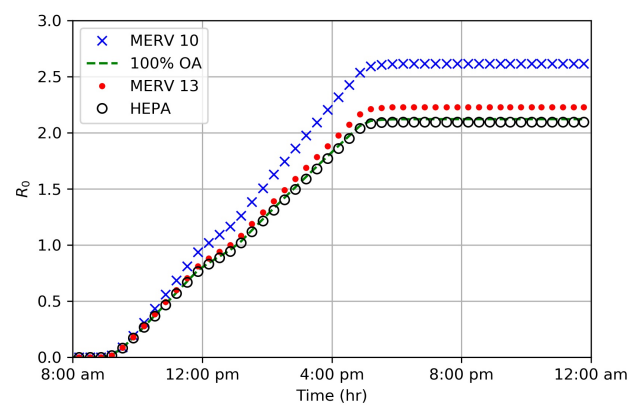

(c) $q=150$ quanta/hr

Figure 11: Predicted number of infections in the worst zone for a hot day with different virus generation rates.

Next, the results for virus concentration in the worst zone for a mild spring day are shown. For this day, all the cases supply 100\% outdoor air 
due to the control of the outdoor air economizer during the mild weather. This causes all the cases to overlap with each other for these plots, since they all essentially supply $100 \%$ clean air to the building.

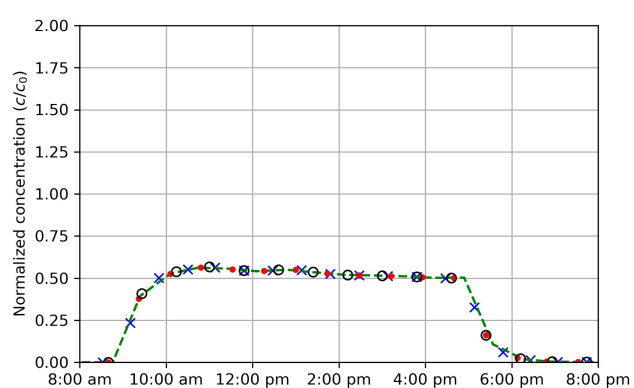

(a) $q=50$ quanta/hr

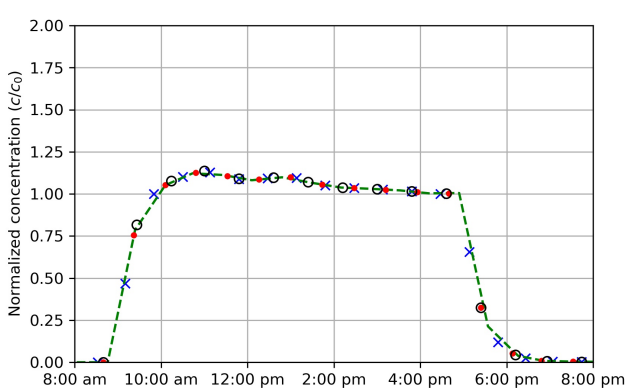

(b) $q=100$ quanta/hr

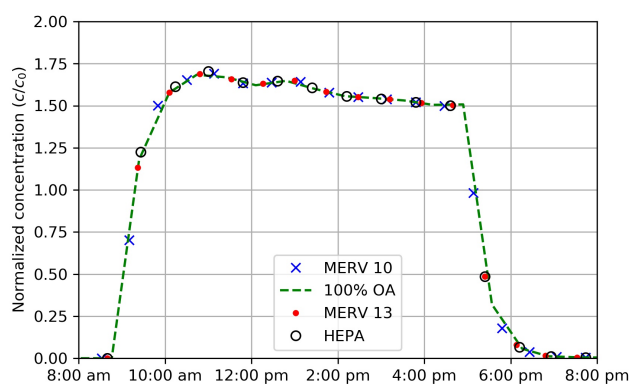

(c) $q=150$ quanta $/ \mathrm{hr}$

Figure 12: Normalized virus concentration in the worst zone for a mild day with different virus generation rates.

The predicted number of infections for this day are shown next in Figure 13. Since the concentrations overlap in Figure 12, it is expected the predicted 
number of infections also overlap for all the cases. Once again, even though these cases supply $100 \%$ clean air throughout the day, it is still likely at least one infection occurs during the day in this zone. The final value of $R_{0}$ for the low generation rate case is still about 0.83 , meaning there is about an $83 \%$ chance one person gets infected.

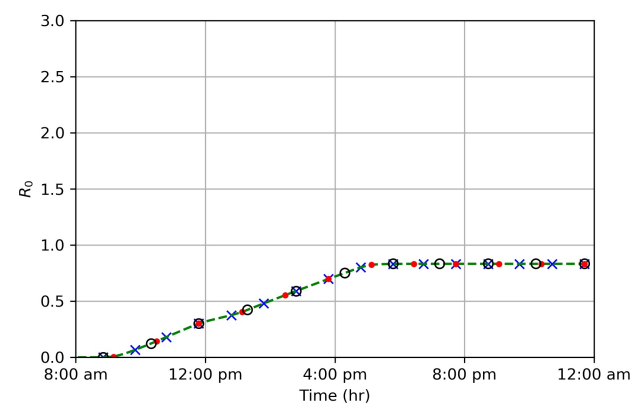

(a) $q=50$ quanta $/ h r$

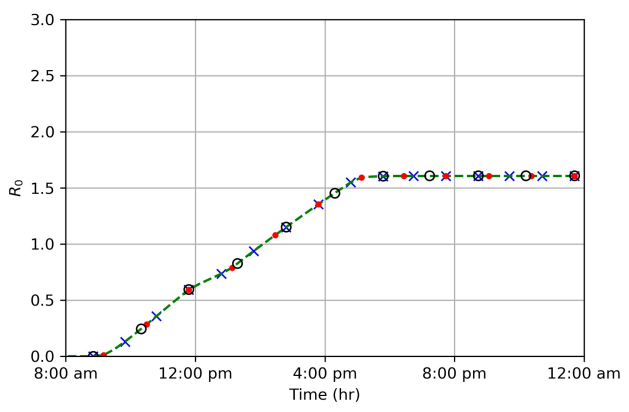

(b) $q=100$ quanta $/ h r$

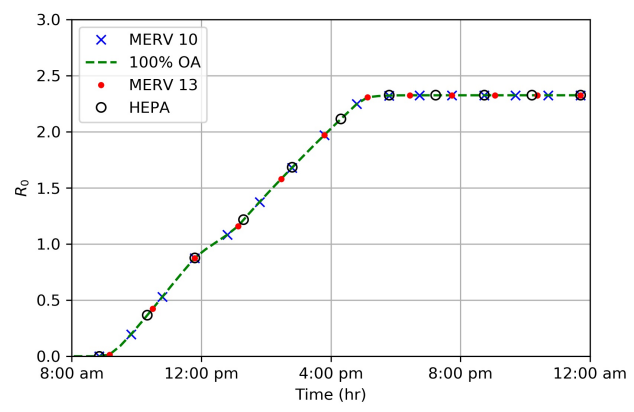

(c) $q=150$ quanta/hr

Figure 13: Predicted number of infections in the worst zone for a mild day with different virus generation rates. 


\subsection{Energy consumption results}

The total annual energy consumption for the four different strategies are shown in Table 2, including the breakdown of energy consumption in terms of fan, cooling, and heating energy. For this HVAC system modeled in Denver's cold climate, the heating energy dominates the total annual energy consumption. Due to the increased system pressure drop, both the MERV 13 and HEPA filter cases use more fan energy throughout the year. However, this increase in energy consumption is negligible compared to the massive increase in energy consumption for the $100 \%$ outdoor air case, mainly due to the significant increase in heating energy. The increases in heating and cooling energy are expected for this case since the system spends more energy to heat/cool the outdoor air, compared to the other cases when the air is recirculated.

A few more subtle annual energy consumption results were found as well. The more efficient filter cases use slightly more cooling energy and slightly less heating energy compared to the MERV 10 case, with these changes more significant for the HEPA filter case. This is due to the fan dissipating thermal energy from the motor to the surrounding air. Since the fan works harder for the efficient filter cases due to the increased pressure drop, it dissipates 
more heat to the supply air, which saves some heating energy but slightly increases the cooling energy throughout the year.

\begin{tabular}{|c|c|c|c|c|}
\hline Case & $\begin{array}{l}\text { Fan Energy } \\
\text { (MWhr) }\end{array}$ & $\begin{array}{l}\text { Cooling Energy } \\
\text { (MWhr) }\end{array}$ & $\begin{array}{l}\text { Heating Energy } \\
\text { (MWhr) }\end{array}$ & $\begin{array}{l}\text { Total Energy } \\
\text { (MWhr) }\end{array}$ \\
\hline $\begin{array}{l}\text { MERV } 10 \\
\text { filter }\end{array}$ & 4.44 & 10.8 & 42.4 & 57.6 \\
\hline $\begin{array}{l}100 \% \\
\text { outdoor } \\
\text { air }\end{array}$ & 4.34 & 12.4 & 102.4 & 119.1 \\
\hline $\begin{array}{l}\text { MERV } 13 \\
\text { filter }\end{array}$ & 4.56 & 10.8 & 42.3 & 57.6 \\
\hline $\begin{array}{l}\text { HEPA } \\
\text { filter }\end{array}$ & 5.87 & 10.9 & 41.3 & 58.1 \\
\hline
\end{tabular}

Table 2: Annual HVAC energy consumption for the different strategies.

The monthly breakdown of energy consumption is shown next in Figure 14 to compare the operational strategies throughout the year. All the cases consume less energy in the warmer months due to the dominance of heating 
energy for this HVAC system and climate. In particular, there is a massive increase in heating energy for the $100 \%$ outdoor air case during the colder months. Interestingly enough, the increase in cooling energy for the $100 \%$ outdoor air case during the summer is much less compared to this increase in heating energy.

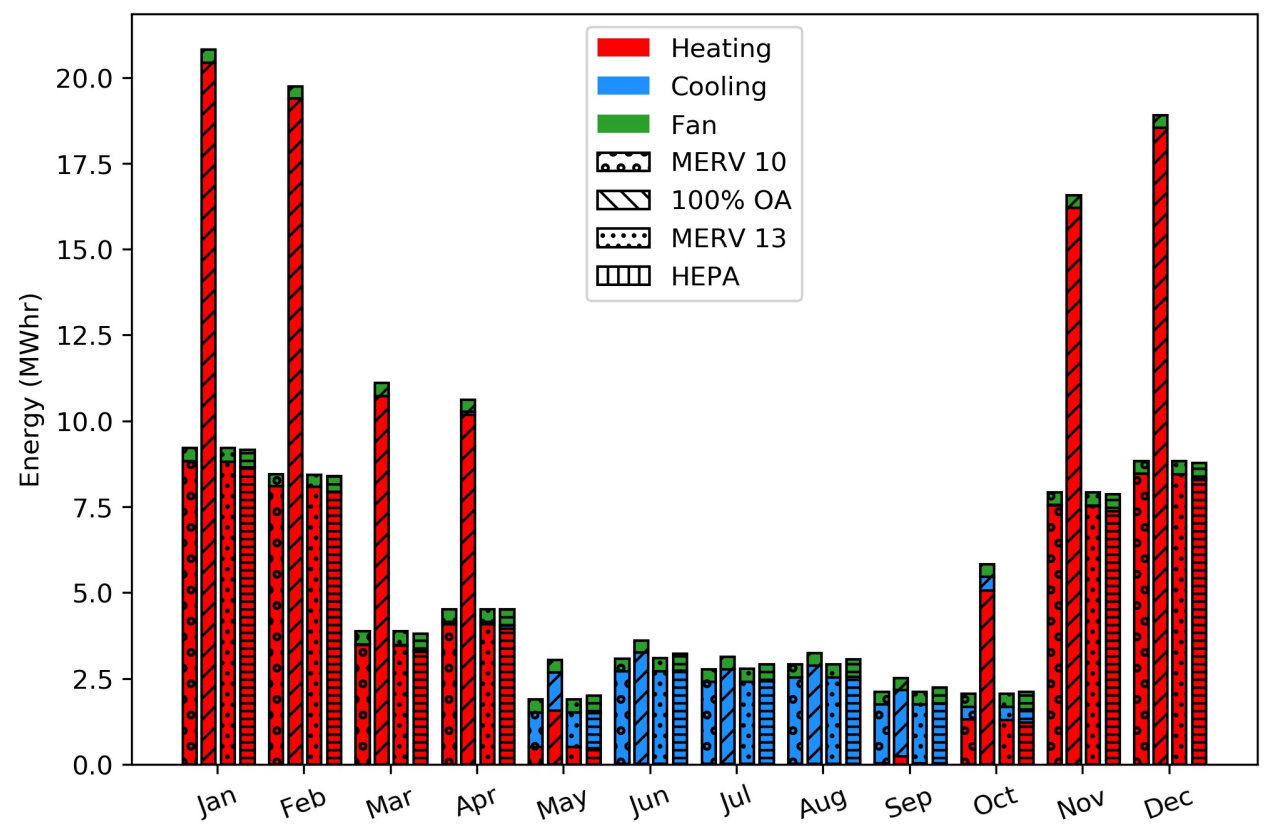

Figure 14: Monthly breakdown of HVAC energy consumption for the different strategies. 


\subsection{Analysis of Combined Results}

Based on the results for virus concentration, use of $100 \%$ outdoor air or HEPA filtration provide the best overall indoor air quality. Although MERV 13 filtration is slightly less effective, it offers similar levels of improvement in indoor air quality with small increases in risk of infection compared to the $100 \%$ outdoor air and HEPA cases. The energy consumption results show similar levels of energy usage for the three filter cases, while the $100 \%$ outdoor air case consumes over $100 \%$ more energy compared to these cases due to the significant increase in heating energy. The final consideration is the cost of the filters. The price of MERV 13 filters can range from $\$ 12-\$ 190$ depending on the depth and style of filter used [5], while HEPA filters can range from \$250-\$350. Based on all these considerations, ASHRAE recommended MERV 13 filtration performs best due to its improvement in indoor air quality with relatively low operational cost. HEPA filtration can be used to provide a slightly safer indoor environment compared to MERV 13 filtration, especially when the system uses the minimum outdoor air fraction, although the cost of the HEPA filter is also higher. 


\section{Conclusion}

Different strategies to improve indoor air quality during the COVID-19 pandemic are investigated for a medium office building in a cold and dry climate. Specifically, the supply of $100 \%$ outdoor air and use of filtration with MERV 10, MERV 13, or HEPA ratings are investigated throughout the year using Modelica-based models. The building is modeled using the Modelica Buildings library and new models for HVAC filtration and transmission of virus are developed to support this study.

The results show the HEPA filter and 100\% outdoor air cases reduce average virus concentration by about 13\% compared to MERV 10 filtration, although the $100 \%$ outdoor air case consumes over $100 \%$ more energy compared to the other cases due to the significant increase in heating energy during the winter months. Use of MERV 13 filtration reduces the average virus concentration by about $10 \%$ and shows similar results for risk compared to the $100 \%$ outdoor air and HEPA cases. However, a HEPA filter can be up to $\$ 328$ more expensive than a MERV 13 filter. Thus, using ASHRAE recommended MERV 13 filtration can achieve a good balance between the indoor air quality and operational cost.

In this paper, we develop computational modules and allow for temporal 
assessment of exposure and risks of indoor occupants. We demonstrate how such an approach allows one to consider the various tradeoffs between exposure risk, HVAC capacity, and energy use. We also show how to consider the marginal benefits of such tradeoffs for current crises and will thus be available if future what-if scenarios are to be considered.

\section{Acknowledgements}

This research was supported in part by the U.S. Defense Threat Reduction Agency and performed under U.S. Department of Energy Contract No. DE-AC02-05CH11231. This work emerged from the IBPSA Project 1, an international project conducted under the umbrella of the International Building Performance Simulation Association (IBPSA). Project 1 will develop and demonstrate a BIM/GIS and Modelica Framework for building and community energy system design and operation.

\section{References}

[1] H. Qian, T. Miao, L. Liu, X. Zheng, D. Luo, and Y. Li. Indoor Transmission of SARS-CoV-2. Indoor Air, 31(3):639-645, 2021.

[2] F.R. Lendacki, R.A. Teran, S. Gretsch, M.J. Fricchione, and J.L. 
Kerins. COVID-19 Outbreak Among Attendees of an Exercise Facility — Chicago, Illinois, August-September 2020. Morbidity and Mortality Weekly Report, 70(9):321-325, 2021.

[3] J. Lu, J. Gu, K. Li, C. Xu, W. Su, Z. Lai, D. Zhou, C. Yu, B. Xu, and Z. Yang. COVID-19 Outbreak Associated with Air Conditioning in Restaurant, Guangzhou, China, 2020. Emerging Infectious Diseases, 26(7):1628-1631, 2020.

[4] Core Recommendations for Reducing Airborne Infectious Aerosol Exposure. https : / / www . ashrae . org / file \% 20library / technical \% 20resources / covid- 19 / core- recommendations- for - reducing- airborneinfectious-aerosol-exposure.pdf, 2021.

[5] T. Ben-David and M.S. Waring. Interplay of ventilation and filtration: Differential analysis of cost function combining energy use and indoor exposure to PM2.5 and ozone. Building and Environment, 128:320-335, 2018.

[6] P. Azimi and B. Stephens. HVAC filtration for controlling infectious airborne disease transmission in indoor environments: Predicting 
risk reductions and operational costs. Building and Environment, 70:150-160, 2013.

[7] L.F. Pease, N. Wang, T.I. Salsbury, R.M. Underhill, J.E. Flaherty, A. Vlachokostas, G. Kulkarni, and D.P. James. Investigation of potential aerosol transmission and infectivity of SARS-CoV-2 through central ventilation systems. Building and Environment, 197:107633, 2021.

[8] Y. Fu, W. Zuo, M. Wetter, J.W. VanGilder, and P. Yang. Equation-based object-oriented modeling and simulation of data center cooling systems. Energy and Buildings, 198:503-519, 2019.

[9] S. Huang, W. Zuo, D. Vrabie, and R. Xu. Modelica-based system modeling for studying control-related faults in chiller plants and boiler plants serving large office buildings. Journal of Building Engineering, 44:102654, 2021.

[10] W. Tian, X. Han, W. Zuo, Q. Wang, Y. Fu, and M. Jin. An optimization platform based on coupled indoor environment and HVAC simulation and its application in optimal thermostat placement. Energy and Buildings, 199:342-351, 2019.

[11] M. Wetter, W. Zuo, T.S. Nouidui, and X. Pang. Modelica Buildings 
library. Journal of Building Performance Simulation, 7(4):253-270, 2014.

[12] M. Wetter, M. Bonvini, T.S. Nouidui, W. Tian, and W. Zuo. Modelica buildings library 2.0. In Proceedings of BS2015: 14th Conference of International Building Performance Simulation Association, 2015.

[13] M. Guo, P. Xu, T. Xiao, R. He, M. Dai, and S.L. Miller. Review and comparison of HVAC operation guidelines in different countries during the COVID-19 pandemic. Building and Environment, 187:107368, 2021.

[14] M. Zaatari, A. Novoselac, and J. Siegel. The relationship between filter pressure drop, indoor air quality, and energy consumption in rooftop HVAC units. Building and Environment, 73:151-161, 2014.

[15] Method of Testing General Ventilation Air-Cleaning Devices for Removal Efficiency by Particle Size. https://www.ashrae.org/File\% 20Library / Technical\%20Resources /COVID-19/52_2_2017_COVID-19_ 20200401.pdf, 2017.

[16] FILTRATION AND DISINFECTION FAQ. https://www.ashrae.org/ technical-resources/filtration-and-disinfection-faq. 
[17] What is a HEPA filter? https://www.epa.gov/indoor-air-quality-iaq/ what-hepa-filter-1, 2019.

[18] Buildings.Examples.VAVReheat. https://simulationresearch.lbl.gov/ modelica/releases/v5.0.0/help/Buildings_Examples_VAVReheat.html\# Buildings.Examples.VAVReheat, 2013.

[19] Commercial Reference Buildings. https://www.energy.gov/eere/ buildings/commercial-reference-buildings.

[20] M. Wetter, J. Hu, M. Grahovac, B. Eubanks, and P. Haves. OpenBuildingControl: Modeling Feedback Control as a Step Towards Formal Design, Specification, Deployment and Verification of Building Control Sequences. In Proceedings of 2018 Building Performance Modeling Conference and SimBuild, 2018.

[21] J. Sines. What is the relationship between pressure drop and flow rate in a pipeline? http://kb.eng-software.com/eskb/ ask- an- engineer / theory- equations- and- calculated-results- questions / relationship-between-pressure-drop-and-flow-rate-in-a-pipeline.

[22] T. Xia and C. Chen. Evolution of pressure drop across electrospun nanofiber filters clogged by solid particles and its influence on indoor 
particulate air pollution control. Journal of Hazardous Materials, 402:123479, 2021.

[23] MERV 10 Pleated Filters. https://www.dwyer-inst.com/PDF_files/ Priced/DF10_cat.pdf.

[24] MERV 13 Pleated Filters. https://www.dwyer-inst.com/PDF_files/ Priced/DF13_cat.pdf.

[25] Engineering Data for Selecting and Using Portable HEPA Filtration Units and Accessories for Nuclear Applications. https://imageserv10. team-logic.com / mediaLibrary / 261 / Engineering_Handbook_rev_5-1311.pdf, 2011.

[26] G. Buonanno, L. Stabile, and L. Morawska. Estimation of airborne viral emission: Quanta emission rate of SARS-CoV-2 for infection risk assessment. Environment International, 141:105794, 2020. 